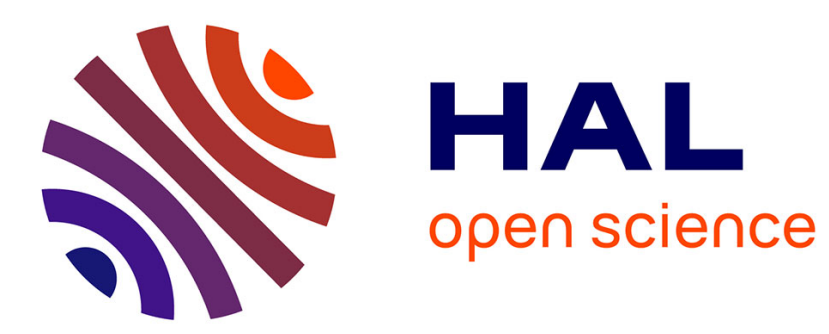

\title{
Le français en Tunisie, enracinement, forces et fragilités systémiques : rappels historiques, sociolinguistiques, et brefs éléments de prospective
}

Francis Manzano

\section{- To cite this version:}

Francis Manzano. Le français en Tunisie, enracinement, forces et fragilités systémiques: rappels historiques, sociolinguistiques, et brefs éléments de prospective. International Journal of the Sociology of Language, 2011. hal-02501361

\section{HAL Id: hal-02501361 \\ https://hal.science/hal-02501361}

Submitted on 6 Mar 2020

HAL is a multi-disciplinary open access archive for the deposit and dissemination of scientific research documents, whether they are published or not. The documents may come from teaching and research institutions in France or abroad, or from public or private research centers.
L'archive ouverte pluridisciplinaire HAL, est destinée au dépôt et à la diffusion de documents scientifiques de niveau recherche, publiés ou non, émanant des établissements d'enseignement et de recherche français ou étrangers, des laboratoires publics ou privés. 


\title{
Le français en Tunisie, enracinement, forces et fragilités systémiques : rappels historiques, sociolinguistiques, et brefs éléments de prospective ${ }^{1}$
}

\author{
Francis Manzano \\ Professeur des Universités \\ Université de Lyon (Lyon-3 Jean Moulin, Centre d'Etudes Linguistiques)
}

\section{Résumé}

La francophonie tunisienne, souvent présentée comme exemplaire (du fait notamment de la francophilie d'une large part de ses élites), s'est historiquement constituée à partir d'un paysage linguistique diversifié. À travers le temps, une capacité à l'adoption de langues extérieures (punique, latin et langues romanes, arabe) peut être constatée à partir d'un fonds local de type berbère. Cette tradition a bénéficié aux langues romanes et notamment au français, connu des élites avant même l'annexion française. C'est pourtant le protectorat français qui, à partir de 1881, créera les conditions de maturation et d'affirmation d'une élite nationaliste tunisienne qui assumera elle-même une sorte d'héritage français, se singularisant ainsi par rapport aux pays maghrébins voisins où les réticences identitaires paraissent à première vue plus vives. Ce qui caractérise en effet le paysage tunisien est l'absence relative de tensions dans les pratiques langagières et le regard que l'on porte sur celles-ci.

La mécanique tripolaire proposée depuis plusieurs années par l'auteur permet de comprendre que la Tunisie se trouve en effet, structurellement, dans une position pionnière par rapport au reste du Maghreb. Si le berbère (l'un des trois pôles) y était encore vivace à la fin du XIXème siècle, il semble se trouver désormais en situation terminale, ce qui distingue encore la Tunisie de ses voisins. De ce fait notamment, l'arabe et le français sont depuis quelques décennies en situation de confrontation directe, contact qui pourrait se densifier et se tendre par la suite, comme le montrent assez bien les évolutions récentes du paysage sociolinguistique (arabisation accrue depuis une dizaine d'années, pressions pour la conversion du français en "langue étrangère », progression symbolique de l'anglais etc.).

\section{Naissance de la francophonie tunisienne, préalables}

D'un point de vue historique et « officiel », la Tunisie est entrée dans l'attraction francophone à la fin du $\mathrm{XIX}^{\mathrm{e}}$ siècle. Les faits sont généralement connus, mais on les rappellera ici brièvement. En réalité, comme on le remarquera à plusieurs reprises, l'attraction linguistique et culturelle avait commencé bien avant et l'on aurait probablement tort de trop focaliser sur l'acte d'annexion politique, même si celui-ci a sûrement déclenché une nouvelle phase dans la relation des langues et des peuples.

À cet égard, bien que peu de chercheurs fassent attention à cette donnée, ou bien qu'ils la considèrent de manière anecdotique, presque toujours superficielle, il faut redire que la Tunisie, bien plus que ses voisins maghrébins immédiats, s'est trouvée constamment exposée à de profonds contacts langagiers et identitaires. Je ne les rappellerai pas tous dans ce cadre, mais ils sont nombreux depuis l'antiquité punique, et probablement avant ${ }^{2}$. Depuis le premier

\footnotetext{
${ }^{1}$ Texte paru dans International Journal of the Sociology of Language (IJSL), pages 53 à 81, numéro spécial The sociolinguistics of Tunisia, coordonné par Lotfi Sayahi. De Gruyter-Mouton, 2011, nº 211.

${ }^{2}$ En termes de chronologie absolue, dès le néolithique l'actuelle Tunisie paraît anthropologiquement (et très vraisemblablement linguistiquement) diversifiée. Un véritable système «tripolaire » se met en place avec la période punique (punique, libyque, pôle « étranger » : grec notamment), qu'il est relativement facile de suivre par la suite (Manzano, 1996), bien que les configurations internes de ce système aient constamment changé, ce qui n'est pas contradictoire de la permanence du système tripolaire lui-même.
} 
millénaire avant J.-C., assurément, la Tunisie n'a jamais été un pays monolingue, et bien au contraire une sorte de laboratoire du contact, phénomène récurrent qui a fondamentalement pétri des personnalités collectives prêtes à admettre la diversité comme une caractérisation normale de leur espace de vie. Voilà pourquoi toutes les langues du paysage tunisien : berbère (ou libyque), punique, latin, variétés romanes (génois, vénitien, catalan...), arabe, italien, français etc. ont toutes possédé une légitimité à des degrés divers, une légitimité admise non seulement par ceux qui peuvent parler ces langues mais aussi par ceux qui ne sont pas amenés à les utiliser régulièrement. Il faut donc bien préciser qu'il s'agit d'une légitimation sociolinguistique et identitaire, car je n'évoque pas ici la légitimation par l'État, quel qu'il soit, autant l'État punique que l'État romain, l'État français (protectorat) ou l'État tunisien bien entendu.

Revenons à l'action de la France. Celle-ci puissance coloniale majeure en Afrique du Nord, se trouve à la fin du $\mathrm{XIX}^{\mathrm{e}}$ siècle dans une logique d'extension et d'affirmation coloniales en Algérie, depuis une cinquantaine d'années. Durant les années 1870-1880 notamment, des efforts sont consacrés en Algérie à la scolarisation des masses autochtones, mais en même temps ces efforts sont durement contrecarrés par le parti des colons (très puissant en Algérie) et profondément défavorable à l'instruction des indigènes.

Dans le même temps, par une logique qui se reproduira partiellement lors de la décolonisation de l'Algérie près d'un siècle plus tard, la partie occidentale de la Tunisie (Monts de Kroumirie notamment) est vue comme une zone politiquement et militairement fragile, une véritable « passoire » où les tribus se déplacent facilement et que les militaires français ont du mal à contrôler. Aux yeux de l'administration coloniale de l'époque, l'agitation répétée sur les marges tunisiennes est en relation au moins indirecte (et peut-être directe) avec les soulèvements et échecs en Algérie. Pour intervenir en Tunisie, la France prendra donc prétexte d'incursions diverses des Kroumirs (ou Khroumirs) tunisiens, en 1881 notamment.

Voici ce qu'écrit Léon Lanier en 1889 :

Au commencement de l'année 1881, l'attitude des tribus tunisiennes de la frontière devint plus hostile. Les actes de brigandage se multipliaient. Des conférences périodiques avaient eu lieu entre les autorités françaises et tunisiennes : mais si elles avaient amené la réparation de certains dommages, elles n'avaient pas rétabli l'ordre ; les Khroumirs, les Ouchteta en étaient venus, grâce à une longue impunité, à exécuter sur notre territoire de véritables razzias.

La campagne militaire dure quelques mois et débouche sur le Traité du Bardo (12 mai 1881). La Tunisie devient alors protectorat de la France. Le système se dédouble en une surface apparente de maintien d'un pouvoir local (en l'occurrence beylical) et la mise en place d'un pouvoir réel de la France à travers la «Résidence générale » et la mainmise sur l'administration et les canaux futurs de la francisation.

Derrière cette surface des événements, on ne perdra pas de vue que l'intervention française se produit dans une période de rivalités accrues entre puissances européennes expansionnistes, au moment où la Tunisie beylicale s'effondre en restant une composante théorique, virtuelle, de la Grande Porte. Dans ce mouvement, l'Italie et la Grande-Bretagne se trouvent en première ligne. Une tentative de mise en place de protectorat anglais en 1873 avait d'ailleurs avorté ${ }^{3}$. Quant aux Italiens, très présents en Tunisie, les entrepreneurs français déjà implantés les considéraient comme des concurrents très actifs à éliminer au plus tôt. Les pouvoirs

\footnotetext{
3 On doit souligner l'intérêt géopolitique évident d'un contrôle de la Tunisie par la Grande-Bretagne, déjà implantée à Malte depuis le début du XIX ${ }^{\mathrm{e}}$ siècle. Quelques années après l'annexion française, 12 à 13000 anglomaltais de Tunisie furent nationalisés français en vertu du droit du sol par un décret du 8 novembre 1921, non sans protestations de la Grande-Bretagne (ECM-Tunisie, 1942, p. 31).
} 
publics français voyaient d'ailleurs avec appréhension un système scolaire italien se confirmer ou se développer. En un mot, du point de vue de la puissance coloniale, les conditions étaient suffisamment pressantes pour que la France intervienne nettement et au plus vite à son profit.

\section{Du passé au présent}

J'ai personnellement beaucoup insisté ces dernières années sur le fait que la francophonie maghrébine méritait des études très fines et qu'on pouvait alors y voir apparaitre des faciès ethno-identitaires et sociolinguistiques. Les attitudes par rapport aux langues en présence et notamment par rapport au français et aux autres langues romanes ne sont pas les mêmes au Maroc, en Algérie et en Tunisie, ce que la plupart des études négligent souvent, se privant ainsi d'un support socio-identitaire important et proposant seulement une sorte de vulgate sociolinguistique essentiellement fondée sur le concept de diglossie, les rapports entre exogène et endogène, national et étranger etc. Or la manière dont nous voyons les langues aujourd'hui est fortement conditionnée par la manière dont nous avons reçu ces langues. Les schématisations évoquées, pour justes qu'elles paraissent, ne sont alors pas vraiment adaptées aux faits.

De ce point de vue, la question des langues apparaît comme relativement détendue en Tunisie, bien plus que chez les voisins maghrébins. C'est un trait frappant. Par exemple, la plupart des Tunisiens interrogés par nos étudiants dans les années 90 disaient ne pas comprendre des attitudes et manifestations ressenties comme violentes chez leurs voisins algériens (défense du berbère, défense du français, réticences ou refus de l'arabisation etc.). Il y avait là quelque chose qui leur paraissait incorrect, incongru. On aurait pu pourtant s'attendre à des propos franchement hostiles soit au français (ex langue coloniale) ou pourquoi pas à l'arabe (puisque les Tunisiens sont généralement réputés bon francophones). Or si de telles opinions apparaissent parfois, elles restent vraiment minoritaires et le plus souvent allusives.

Autre exemple : alors que l'Italie a joué un rôle important et aurait pu devenir assez logiquement une puissance coloniale dans cette région (on vient de le voir) ${ }^{4}$, d'autant que beaucoup de groupes d'origine italienne (souvent sicilienne) étaient implantés sur les côtes notamment, le poids linguistique et culturel de l'italien a été minimisé, et il l'est toujours dans la plupart des discours. On s'aperçoit en écoutant les gens, que l'italien est une langue qui ne « compte » pas vraiment, on le relègue ainsi à des fonctions assez marginales et utilitaires : c'est ainsi une langue qui permet d'avoir des nouvelles de l'Europe et du monde par la RAI ${ }^{5}$, ou c'est encore une langue qui permettra éventuellement d'immigrer en Europe ou de recevoir des touristes d'origine italienne (encore relativement nombreux) etc. On se trouve bien dans une logique de «marché », une perception purement marchande et par nature fort peu agressive des langues.

Autre exemple encore. Tous les Maghrébins, d'un bout à l'autre, sont attirés de longue date par la langue anglaise. En cela, ils illustrent un mouvement mondial, mais aussi une logique de compétition des langues européennes en Méditerranée (suggérée plus haut). Ce que l'Angleterre et l'anglais n'ont pas réussi en 1873 pourrait être à présent réalisé par d'autres canaux. En fouillant un peu ce dossier, on se rend compte que la Tunisie (à travers ses élites et

\footnotetext{
${ }^{4}$ Il faut rappeler qu'au XX ${ }^{\mathrm{e}}$ siècle, l'Italie, pour sa part, n'a jamais cessé de voir la Tunisie, ainsi que la LibyeCyrénaïque, comme autant de prolongements presque naturels de l'Italie, ce qui est géographiquement et humainement compréhensible compte-tenu de relations extrêmement anciennes et jamais interrompues entre les deux zones. La version dure de cette relation apparut évidemment avec le fascisme italien, qui jugea l'ensemble évoqué comme revenant de droit à l'Italie, ce qui n'est pas exactement la même chose.

${ }^{5} \mathrm{La}$ télévision italienne était facilement reçue à la fin du $\mathrm{XX}^{\mathrm{e}}$ siècle (RAI 1 surtout, voire RAI 2, mais aussi diverses autres chaînes régionales italiennes ou siciliennes).
} 
ses pouvoirs publics) s'est montrée pilote dans ce domaine. La mécanique qui sous-tend le raisonnement est la suivante.

Plus que leurs voisins, les Tunisiens semblent avoir conscience qu'ils ne peuvent se dispenser d'une langue de grande diffusion, ce que l'on peut dénommer langue d'ouverture (d'où la relative décrispation évoquée). C'est encore très logique dans un contexte de «marché ». Le français a joué ce rôle jusqu'à présent, mais on sent bien qu'une partie des intellectuels notamment, tout en montrant des formes diverses d'attachement au français, se révèle favorable à l'idée que l'anglais pourrait à terme remplacer le français dans ses fonctions internationales. Des expériences pédagogiques diverses pourraient être citées, mais pour l'instant l'anglais ne parvient pas à prendre pied fermement dans le système scolaire tunisien autrement qu'en tant que langue seconde, ce qui ne signifie pas qu'il ne puisse concurrencer à terme le français. Tout semble donc se passer comme si on se préparait calmement, mais depuis plusieurs années, à un changement (éventuel) de langue d'ouverture. Cela pourrait se faire comme ne pas se faire, mais si cela se fait, cela devrait se faire probablement sans états d'âme.

Dernier exemple enfin. Il concerne le berbère. Cette langue peut être considérée dans sa phase terminale au cours du $\mathrm{XXI}^{\mathrm{e}}$ siècle. Les derniers locuteurs sont massivement des diglottes, répartis dans quelques villages du sud tunisien et dans une partie de l'île de Djerba. Quand on connaît les pays voisins du Maghreb (Algérie, Maroc), on ne peut qu'être frappé en même temps par les disproportions des langues en présence, et par la discrétion qui entoure ici le berbère. Pour les données on évoque depuis plusieurs années une proportion de $1 \%$, ce qui est fort peu et accrédite l'idée d'une prochaine disparition de la langue. Quant à l'environnement sociolinguistique, on ne retrouve à peu près rien des passions, des conflits et des mécaniques d'affirmation qui entourent la question du berbère en Algérie et au Maroc. Le berbère s'éteint calmement, et il m'est arrivé d'écrire à ce sujet que tout se passait comme si le berbère de Tunisie n'était plus visible, se trouvant placé dans une sorte de transparence fantomatique qui le consacre comme déjà mort, alors que quelques milliers de locuteurs s'en servent encore. Il faut souligner que cette évacuation sociolinguistique d'ensemble s'accompagne d'une déviation et dilution pragmatique dans le langage géographique et culturel ambiant puisqu'on évoque très couramment en Tunisie les «Berbères », mais pour désigner essentiellement les campagnards face aux urbains. Évidemment cette pratique, qui s'incruste dans les cerveaux, ne peut que noyer plus encore les derniers Berbères de Tunisie (locuteurs de « chelha ») dans une masse indistincte.

Il me semble donc que les trois dossiers qui viennent d'être brièvement évoqués, quel que soit l'angle d'attaque de la question des langues en usage en Tunisie, montrent qu'on a très peu de chances de rencontrer des avis entiers, voire totalitaires. Généralement, pour tenter d'obtenir de tels propos en Tunisie, il faut introduire une certaine dose de provocation. Encore n'est-on pas assuré de réussir, alors qu'en Algérie ou au Maroc, toute enquête épilinguistique révèle très rapidement des positionnements tranchés, eux-mêmes volontiers provocants ${ }^{6}$.

Bien entendu, on pourrait mettre de telles attitudes sur le compte soit du caractère « raisonnable» des informateurs tunisiens, soit encore d'une prudence ou d'une habileté liée au contexte socio-culturel et politique tunisien. Mais de telles explications paraîtraient un peu courtes du point de vue d'une sociolinguistique fondamentale. C'est plutôt du côté de la constitution du paysage sociolinguistique qu'il faut chercher des éléments de réponse.

On peut, en l'adaptant, évoquer le concept de loyauté linguistique (Weinreich), mais à condition de l'évoquer peut-être de manière négative. Si un tel concept paraît assez performant

\footnotetext{
${ }^{6}$ Manzano (1993).
} 
pour nombre de Marocains ou Algériens ${ }^{7}$, force est de constater qu'il n'est guère valable en Tunisie, y compris même pour une langue comme l'arabe qui est à présent, manifestement, la langue réelle de la quasi-totalité des Tunisiens. Au moins sur ce plan on pourrait s'attendre à ce que des engagements très clairs soient réalisés dans cette direction.

\section{Retour au protectorat}

Après la mise sous tutelle politique, l'instruction française avait semble-t-il commencé par concurrencer un système d'instruction italien bien implanté dans la région de Tunis/La Goulette, mais aussi dans la plupart des villes importantes du littoral (Sousse, Sfax) où les populations d'origine italo-romane constituaient la première communauté européenne $\left(\right.$ Manzano, 1996) ${ }^{8}$. Néanmoins, dès le milieu du XIX ${ }^{\mathrm{e}}$ siècle, les congrégations religieuses à base française étaient actives en Tunisie ; en cela elles préparèrent efficacement le terrain de la francisation ultérieure (Soumille, 2000 ; Naffati \& Queffélec, 2004).

Différents actes non seulement symboliques mais pratiques du protectorat peuvent être observés et soulignés par la suite, comme la suppression de l'enseignement de l'italien et du $\operatorname{turc}^{9}$ au sein du fameux collège Sadiki, pépinière connue des élites dirigeantes tunisiennes. Il fallait ainsi trancher avec le passé « turc » de la Tunisie, en signifiant de nouvelles directions pour les élites cultivées du pays.

On peut légitimement penser que la francisation, appuyée sur le protectorat, a pu donc capter dans ce pays une dynamique précédemment favorable à l'italien et aux langues étrangères en général, prolongeant et récupérant à son profit une nette et ancienne tradition d'ouverture et de circulations culturelles évoquée au début de l'article.

Quoiqu'il en soit, quelques années après le début du protectorat (1898), 6\% des petits Tunisiens en âge d'être scolarisés semblent avoir été, à des degrés divers sans doute, exposés au français. Le chiffre peut paraître maigre à première vue, mais il est probablement révélateur d'une originalité tunisienne dès cette époque si on le rapporte aux structures socioéconomiques et culturelles du Maghreb. Celui-ci est alors profondément rural et si l'on compare avec la puissance colonisatrice elle-même, on doit rappeler que le nouvel enseignement laïc et obligatoire de France rencontre au même moment des difficultés à plus d'un égard comparables dans la France paysanne du temps, notamment un absentéisme problématique des jeunes ruraux happés par des temps forts agricoles et retirés de l'école en fonction des besoins des familles ${ }^{10}$. La Tunisie des champs n'a pas échappé à ce type de contrainte, et par conséquent l'efficacité de la scolarisation. C'est donc cette contrainte sociale qui rend d'autant plus intéressant le score observé.

Celui-ci doit surtout être rapproché de chiffres bien plus mauvais concernant l'Algérie de la même période, ainsi que le fait remarquer Pierre Foncin (1900) au début de cet extrait :

La proportion est de 6 sur 100 (en Algérie elle est de 3 sur 100 seulement et la conquête de l'Algérie est achevée depuis près de 50 ans, tandis que le protectorat de la Tunisie n'a pas vingt ans d'existence).

\footnotetext{
${ }^{7}$ Lesquels s'engagent assez facilement pour l'arabe/l'arabophonie, le berbère/la berbérophonie, le français/la francophonie (voire deux ou toutes ces paires), ce en quoi on peut considérer qu'ils font effectivement preuve de loyauté linguistique, dans un sens ou dans l'autre.

8 Jusqu'aux années 1880, au sein même des établissements religieux français (qui existent déjà), les jeunes Italiens (et Maltais) sont nettement majoritaires : plus de $90 \%$ en général (Soumille, 2000). Au début du protectorat les Français ne représentent que $4 \%$ de la population « européenne », contre $59 \%$ pour les Italiens et $37 \%$ pour les Anglo-Maltais (Martin, 1993, cité par Naffati \& Queffélec, 2004).

${ }^{9}$ Au bénéfice du seul français pour les langues étrangères (Sraieb, 1995 ; Queffélec \& Naffati, 2004).

${ }^{10}$ Ces phénomènes, très pesants durant le troisième quart du XIX ${ }^{\mathrm{e}}$, sont une des sources des lois scolaires de la fin du siècle, notamment la loi du 28 mars 1882 dont plusieurs articles (notamment 7 à 15) tournent autour des absences, sanctions et dispenses.
} 
La statistique des élèves au point de vue des nationalités montre qu'aucune race n'est réfractaire à l'instruction.

Garçons: Français, 1576 ; Musulmans, 4100 ; Israélites indigènes, 2424 ; Italiens, 1696 ; Maltais, 783 ; Divers, 126.

Filles : Françaises, 1239 ; Musulmanes, 31 ; Israélites indigènes, 1298 ; Italiennes, 1362 ; Maltaises, 709 ; Diverses, 97.

Il n'y a d'exception que pour les jeunes Musulmanes, qu'un antique préjugé tient séquestrées et prive des bienfaits de l'école. Peut-être faudrait-il commencer par les instruire à domicile (p. 128).

La dernière remarque, clairement justifiée par les données, fait apparaitre un caractère « maghrébin » plus que spécifiquement « musulman » ${ }^{11}$. On remarquera en effet que, tout en étant moins accusée, la tendance à garder les filles à la maison est également marquée chez les «Israélites indigènes », que l'on évoquera plus loin. Il n'y a pas non plus égalitarisme strict chez les Romans (Italiens notamment) ou les Maltais ${ }^{12}$, mais se marque donc bien ainsi une opposition comportementale binaire non-maghrébin vs. maghrébin, qui se retrouve aussi en Algérie et au Maroc.

À ce propos, on ne serait pas complet si l'on n'observait que le système scolaire français se pose, en Tunisie et au Maghreb, comme le seul véritablement digne de ce nom, minimisant d'emblée le système arabo-musulman de l'école coranique, système perçu comme un catéchisme anachronique et parfois fanatique ${ }^{13}$, non comme une école. Voici ce qu'écrivait encore Pierre Foncin (1882) à propos des écoles dites alors « du Coran », ou «Koran » :

Sous la direction d'un vieillard impassible armé d'une longue baguette, des enfants accroupis presque tout le jour dans une chambre basse crient à tue-tête ; c'est un vacarme assourdissant, chacun d'eux a sur les genoux une planchette sur laquelle sont inscrits des versets du Coran; il s'époumone à les répéter sans en comprendre un mot, jusqu'à ce qu'il les sache imperturbablement par cœur. Ce genre d'étude n'appartient à aucune classification connue; il n'offre aucun danger politique, mais il est aussi contraire à l'hygiène qu'abêtissant. ${ }^{14}$

\section{Facteurs de la pénétration}

La Tunisie, comme le Maroc eut donc le statut de protectorat. Aussi peut-on trouver quelques points communs dans la francophonie de ces deux pays. Mais s'il existe une différence palpable entre les deux paysages linguistiques marocain et tunisien, c'est probablement en termes de pénétration et de diffusion de la langue française qu'il faut l'apprécier, pénétration plus profonde en Tunisie, bien que fondamentalement les mécanismes objectifs en soient très proches.

Avec un peu de recul, cette relative facilité d'installation du français, amplement vérifiée par la suite, est à mettre en rapport avec les considérations plus haut développées. Pour les résumer, on pourrait dire que l'Histoire profonde de la Tunisie a préparé cette région du

\footnotetext{
${ }^{11}$ Cette qualification globalisante de «musulmans» gomme donc l'opposition intra-maghrébine arabophones vs. berbérophones (voir ci-après).

${ }^{12}$ La Sicile et Malte se trouvent à moins de 100 kilomètres des côtes tunisiennes. Il faut rappeler que les Maltais (que l'on retrouve en Algérie) proviennent d'un archipel où l'arabe est langue natale de nombre d'habitants. Mais en outre, l'italien [qui a fortement marqué le lexique maltais] et une autre langue européenne (l'anglais) ont provoqué une forte rupture diglossique dans le paysage sociolinguistique (maltais vs. langue européenne), rupture certainement accentuée par le fait que les Maltais sont massivement catholiques. Tout cela explique leur position très particulière dans le Maghreb colonial et leur très bonne intégration d'ensemble aux régions colonisées de l'Est du Maghreb.

${ }^{13}$ De même que dans la France de ce temps, toute charge contre l'enseignement religieux est susceptible de compléter une idéologie républicaine de la scolarité laïque et obligatoire.

${ }^{14}$ Cette vision française, tout en se maintenant pratiquement jusqu'à nos jours chez des Maghrébins mais sous d'autres formes, perdit évidemment de sa violence en avançant dans le $\mathrm{XX}^{\mathrm{e}}$ siècle. Les formulations ont changé, mais ce type de constat s'entend toujours.
} 
monde méditerranéen à concevoir le contact des langues et des identités comme ordinaire, c'est-à-dire dans l'ordre normal des choses.

L'installation du français est aussi à mettre en rapport avec un système d'enseignement probablement plus ancien, plus ouvert et plus complet qu'ailleurs, avant même l'installation de la France. Rappelons ainsi que le collège Sadiki, évoqué plus haut et si important pour l'élite tunisienne, avait été fondé en 1875 par un Premier ministre du bey. Le protectorat n'eut donc en réalité qu'à conformer et contrôler la dynamique de cet établissement, très moderne pour l'époque. Le tri entre le peuple et les élites étant déjà assuré en amont du protectorat, il n'y eut pas de discontinuité fondamentale et, au contraire semble-t-il, ces préalables donnèrent au français, langue de culture et d'ouverture déjà appréciée (comme plus généralement au sein des élites méditerranéennes), une forme de légitimité en tant que langue étrangère privilégiée d'une élite sociale dès ce moment tournée vers l'Europe ${ }^{15}$.

Dans cet ordre d'idée, il n'est pas inutile de souligner que lorsque commença le protectorat français en Tunisie la pénétration scolaire et culturelle du français s'était elle-même affirmée depuis deux ou trois décennies dans le monde ottoman, notamment après la guerre dite « de Crimée » (1853-1856). Différents établissements primaires et secondaires furent fondés en Turquie et dirigés par des « frères » et congrégations d'origine françaises. On peut également signaler que depuis 1868 l'enseignement était donné en français pour les matières scientifiques au lycée de Galatasaray (Constantinople).

Cet état de fait ne pouvait être sans incidence sur les élites tunisiennes dès avant le protectorat, comme le soulignera le Président Bourguiba dans les années 60.

\section{Sur le protectorat : brèves remarques sur l'organisation et les incidences linguistiques $^{16}$}

Le premier point frappant est l'absence de volonté apparente de la France de transformer en bloc et d'office les sociétés locales, même si la puissance étrangère régit de fait l'ensemble de l'édifice politique. Dans les faits, le Résident Général contrôle l'ensemble du système, organisé en trois cercles. Le premier cercle, au sommet de la pyramide, est celui du contrôle politique français, différents services de la Résidence Générale, Secrétariat Général, véritable gouvernement dont émanent les directives et orientations politiques principales. A l'autre bout, un cercle tunisien d'administration directe en quelque sorte (Premier Ministre, caïds, cheikhs, magistrats tunisiens etc.). Entre les deux, la dynamique même du protectorat tendit très rapidement à développer une administration moderne et technique de type français, fondée sur la langue et les savoirs techniques et administratifs de la France. C'est ce cercle qui a transposé en Tunisie les matrices françaises des grands ministères et administrations, où l'on retrouve bien entendu des directions: de l'Instruction publique et des Beaux-Arts, des Affaires Économiques, des Mines, de l'Office des Postes et Télégraphes etc. Ce cercle intermédiaire constitua en réalité le cœur de la machine de francisation, d'autant que le cercle «tunisien » parut vite pour la plupart des acteurs un cercle à peu près totalement inféodé au précédent, dominé du moins, limité à l'exécution de directives définies ailleurs, cantonné en outre à des domaines et compétences limités comme la justice, la religion, le droit musulman (par exemple la gestion des biens « habous »).

Outre l'administration, un autre levier de francisation fut évidemment celui de l'enseignement. Le système fut fractionné logiquement en :

\footnotetext{
${ }^{15}$ Mais c'est dans ce même canal que seront formées très logiquement (et comme semblaient le redouter les autorités du protectorat) les élites nationalistes du $\mathrm{XX}^{\mathrm{e}}$ siècle.

${ }^{16}$ Sans que l'on puisse parler d'identité parfaite, les principes généraux du protectorat tunisien se retrouvent en bonne partie appliqués au Maroc, où ils furent d'ailleurs radicalisés et systématisés par la politique du Général Lyautey, premier Résident Général au Maroc, à partir de 1912.
} 
(a) un sous-système français avec écoles et lycées français. Ce système principalement destiné aux jeunes Français, reçut aussi des Italiens, des Maltais, des Israélites, des élèves musulmans également ${ }^{17}$.

(b) un sous-système mixte, dit généralement «franco-arabe » (enseignement bilingue, mais avec domination horaire du français sur l'arabe), exclusivement destiné aux musulmans.

À côté de ces deux grandes options se maintient un sous-système traditionnel ou fondamental, fondé sur un réseau d'écoles coraniques (établissements primaires). Cet ensemble, par définition, fonctionne sur des bases arabophones et islamiques, bien que des tentatives soient apparues pour y introduire quelques éléments de français et de sciences profanes ${ }^{18}$. Il comporte une structure supérieure avec la Grande Mosquée de la Zeitouna ou Zitouna de Tunis, centre célèbre de formation des élites coraniques avant, pendant et après le protectorat. De nombreux acteurs du cercle tunisien plus haut évoqué (magistrats, dignitaires divers) avaient suivi cette formation.

On évitera d'oublier le versant professionnel de cet enseignement du protectorat. Ce fut en effet un élément important du dispositif français, pas seulement en Tunisie mais dans l'ensemble du Maghreb. Ainsi que je l'ai rappelé il y a peu (Manzano, 2007), on peut y voir la trace d'un effort plus général de l'instruction française pour engager les jeunes des classes populaires et rurales dans une formation professionnelle dont le but général était d'éviter de former des déclassés, facteurs de trouble politique potentiel. Mais on doit aussi comprendre qu'un tel système, par la force des choses, fut destiné presque exclusivement au peuple tunisien ainsi marginalisé de facto par rapport aux précédentes options.

Ces données rapidement synthétisées nous permettent de comprendre que nous sommes en présence d'un système plutôt efficace de séparation des identités, des langues et des pouvoirs, mais qui devait secréter automatiquement des frustrations. Il y a donc par suite dédoublement structuré de l'administration (Musulmans vs. Européens), de la justice (droit musulman vs. droit français ou international), et même de l'habitat puisque sont créées au cours du protectorat des «villes nouvelles » (vs. «médinas ») qu'habiteront préférentiellement des Français ou Européens puis, progressivement, des franges de francisés partiels.

Pour finir, il faut envisager le cas du culte musulman durant cette période, car c'est un fondement essentiel du pôle «arabo-musulman». On doit à ce propos souligner que de nombreux hommes politiques français de la première partie du $\mathrm{XX}^{\mathrm{e}}$ siècle sont convaincus que la France est désormais une puissance «musulmane». Beaucoup sont au pouvoir, au Maghreb notamment, et dans leur esprit le noyau musulman (ou arabo-musulman) doit être en même temps respecté et canalisé. L'administration française n'intervint donc pas directement dans ce domaine, en le traitant à distance et en le contrôlant étroitement aussi bien par les moyens classiques (police, renseignement, partis politiques) que par un système d'acteurs tunisiens relais entre la composante islamique et la dynamique française du système général. On a déjà vu qu'un système d'enseignement arabo-musulman fut toujours maintenu, dans lequel le protectorat français n'intervenait pas, une sorte de domaine réservé.

Mais on ne peut s'empêcher de considérer cela sous un autre angle. À un moment où la laïcité, la séparation des Églises et de l'État se précisaient en France, le protectorat de la Tunisie (comme celui du Maroc) vit en réalité trois «Églises » principales ${ }^{19}$ s'afficher plus nettement et se structurer pour exister. On comprend que cette « séparation » bénéficia d'abord aux deux

\footnotetext{
${ }^{17}$ Depuis l'indépendance de la Tunisie, ce sous-système a été prolongé par les établissements français de la Mission Culturelle Française (écoles, collèges et lycées). De tels établissements jouent bien-sûr un rôle important dans la perpétuation de la francophonie tunisienne.

${ }^{18}$ D'après R. Le Tourneau (Directeur Général de l'Instruction Publique), dans ECM-Tunisie, p. 108.

${ }^{19}$ Correspondant aux trois grandes religions monothéistes de la Méditerranée.
} 
communautés : chrétienne et israélite, même si les musulmans dans leur ensemble semblent avoir été assurés du respect de leur identité, bien plus fortement qu'en Algérie par exemple.

En outre, le fait de fournir une assise et une reconnaissance institutionnelle à deux religions précédemment dominées et statistiquement minoritaires dans le monde maghrébin musulman $^{20}$, favorisa le rapprochement linguistique (sur la base du français) de deux communautés d'origines et de fonctionnements très différents. C'est une question assez complexe qu'on ne peut qu'évoquer brièvement, mais qu'il faut avoir présente à l'esprit.

Les Catholiques sont pratiquement tous d'origine européenne ou maltaise. À différentes reprises cette communauté euro-chrétienne elle-même composite, se révélera antisémite par ses attitudes et ses rejets, particulièrement dans les périodes de tension et autour notamment des questions d'attribution de la nationalité française à des communautés d'origine tunisienne, sans compter la période vichyste lors de la seconde guerre mondiale ${ }^{21}$.

Chez les Juifs existait en revanche une composante numériquement majoritaire de Juifs d'origine maghrébine et tunisienne en l'occurrence, composante inscrite dans le tissu langagier tunisien (communautés arabophones ${ }^{22}$ ou berbérophones). À cette base se sont adjoints à plusieurs reprises des Juifs anciennement originaires d'Europe (Andalous, Livournais etc.) ou venus de France lors du protectorat français ${ }^{23}$, non sans chocs bien connus et parfois même séparations pures et simples des sous-communautés ${ }^{24}$.

Diverses puissances coloniales, dont la France, se posèrent au cours du XIX ${ }^{\mathrm{e}}$ siècle comme protectrices à des degrés divers de ces communautés juives d'Afrique du Nord. Une certaine francophilie s'était avérée dès le début du siècle chez nombre de Juifs maghrébins, fondée sur l'idéologie égalitaire révolutionnaire, la vive impression procurée par la période napoléonienne en Méditerranée etc. La diplomatie française intervint aussi directement, notamment en 1857, dans l'affaire Batou $\mathrm{Sfez}^{25}$. Sa pression, conjuguée à celle de l'Angleterre, conduisit le pouvoir du Bey à améliorer le sort des communautés juives tunisiennes (suppression du statut ancien de dhimmi, égalité juridique officielle etc.). Enfin, dans cette quête des juifs tunisiens pour se « soustraire à la domination des Arabes musulmans auxquels ils avaient été assujettis depuis la conquête de l'Afrique du Nord » (Hagège \& Zarka, 2001-9), la période du protectorat donna différents arguments et signes, sans procéder à une nationalisation en bloc, comme ce fut le cas en Algérie. Elle permit surtout à des organisations d'origine juive françaises (Alliance Israélite Universelle ${ }^{26}$ ), à différentes sociétés et

\footnotetext{
${ }^{20}$ Dans l'ensemble arabo-musulman et notamment au Maghreb, les communautés israélites ou chrétiennes disposaient traditionnellement du statut de dhimmi ou dhimmis, accordé aux « Gens du Livre ». Sous réserve d'un impôt (la jizia ou djizia) et à la condition de ne pas faire preuve de de prosélytisme, la pratique de ces cultes communautaires et de langues particulières était admise. Mais différents interdits sociaux et professionnels, d'assez nombreuses contraintes immobilières, vestimentaires etc. montrent toutefois qu'il s'agissait bien d'un statut peu enviable sociologiquement (Sebag, 1991; Taïeb, 2003). Les Chrétiens, par le très ancien système des comptoirs ou fondouks des différentes nationalités européennes (zones réservées aux commerçants européens et disposant de leurs propres chapelles), échappaient partiellement à cette minoration culturelle et sociale.

${ }^{21}$ L'attribution de la nationalité française aux Juifs algériens (« décret Crémieux », 1870), inspira certainement des positionnements tranchés, et déclencha une grande méfiance tant au sein de communautés juives traditionalistes (aux yeux desquelles la francisation signifiait à terme une perte de l'identité originelle) qu'au sein d'une large composante française (pour qui, en simplifiant, l'on prenait ainsi le risque de judaïser la société française).

${ }^{22}$ Que l'on qualifie assez régulièrement de « judéo-arabes ».

${ }^{23}$ Différents militaires, administrateurs, enseignants.

${ }^{24}$ Les différentes sources utilisées pour cette communauté s'accordent toutes sur la séparation des Livournais et des Tunisiens (judéo-arabes). Une séparation qui incluait les codes vestimentaires, les lieux de prière etc.

${ }^{25}$ Juif tunisien exécuté car accusé d'avoir insulté le Prophète Mohamed lors d'une altercation.

26 Fondée en 1860, l'AIU créa différents établissements dispensant un enseignement moderne de type francophone. Parmi ses premiers présidents on trouve Adolphe Crémieux, dont le nom reste attaché au décret cité plus haut.
} 
mouvements favorables à la francisation $^{27}$ de fournir le cadre général d'une « occidentalisation » croissante et, pour finir, le déport majoritaire par rapport au socle judéotunisien.

Dans les faits pourtant, non seulement la religion mais les pratiques ethniques ou langagières séparaient en théorie les communautés chrétienne et juive, et l'on évitera de considérer que le protectorat français a définitivement rapproché ces communautés. D'un côté, la première allait logiquement et pas à pas vers la francisation, organisée au fond par une romanité commune et une large communauté de pensée sur fond de communauté de religion et de culture, au-delà des divergences sociales et régionales ou politiques certes nombreuses. Pour la seconde, en revanche, s'est posé très vite, dès avant le protectorat, mais surtout pendant le protectorat, la question fondamentale de savoir si la communauté devait assurer son avenir sur la base de son socle tunisien (culture juive ou judéo-arabe, au fond composante normale du tissu tunisien), ou se retrouver dans une nouvelle spirale identitaire et surtout sociolinguistique, d'attraction française. Cette intégration traumatique a certainement constitué l'un des conditionnements de l'œuvre d'Albert Memmi, d'origine tunisienne (1957, 1962, 2004).

C'est la voie de la francisation qui devait pourtant l'emporter malgré nombre de réticences souvent exprimées, conduisant à un déplacement symbolique global des groupes juifs de Tunisie vers la francité, suivi d'un déplacement physique à peu près complet (exode) vers la fin du $\mathrm{XX}^{\mathrm{e}}$ siècle. Pour autant, et jusque sous nos yeux, bien que se réduisant sans cesse, une composante judéo-arabo-berbère s'est maintenue en Tunisie ${ }^{28}$.

Que faut-il donc retenir du protectorat?

Sur le plan sociolinguistique et identitaire, les disproportions sont évidentes. Le français, langue exogène, expression d'un pouvoir colonial, occupe les positions principales (administration, enseignement, presse etc.). Pour autant, réduire le français au statut de langue coloniale pure ne conviendrait pas, cette langue bénéficiant dès avant le protectorat d'un statut symbolique haut. Le protectorat allait donc cumuler cette puissance symbolique de la langue française et une officialité croissante démontrée par les multiples capacités moderne du français mises en lumière par ce que l'on considérait à l'époque comme une incapacité fondamentale de l'arabe à investir la modernité. La machine ainsi installée allait conduire à intégrer, à des rythmes différents, plusieurs groupes périphériques européens, romans, mais aussi une bonne part de la communauté juive régionale, en commençant par le haut, par les élites donc, un processus qui peu à peu allait toucher la plupart des membres de cette communauté.

Pour la composante arabo-musulmane (majoritaire), voire berbère et musulmane (elle-même déjà minoritaire), le protectorat fut en même temps prudent et radical.

L'approche est prudente, car on ne voit pas d'attaque frontale durant cette période. Ce serait même plutôt le contraire. Une considération certaine existe pour l'arabe, les orientalistes sont nombreux, ont des positions officielles parfois importantes. Mais cette considération généralement positive vaut surtout pour l'arabe langue littéraire et cultuelle, c'est-à-dire une langue par ailleurs vue comme coupée des masses tunisiennes et donc d'un très faible rendement sociolinguistique ${ }^{29}$. Une langue symboliquement forte mais qui ne pourrait presque jamais être substituée au français dans le monde moderne.

\footnotetext{
${ }^{27}$ Comme le mouvement moderniste autour du journal francophone La Justice (Hagège \& Zarca, p. 21).

${ }^{28}$ La plupart des Juifs de Tunisie ont émigré vers la France, Israël et dans d'autres pays, suivant en cela la dynamique de la décolonisation. D'après certaines sources, il resterait aujourd'hui 1700 Juifs en Tunisie, principalement localisés à Tunis et dans l'île de Djerba. Après la seconde guerre mondiale (les données restent sujettes à caution, ce sont des estimations) ils étaient probablement entre 60.000 et 120.000 .

${ }^{29}$ Bien que les linguistes s'intéressent à l'arabe dialectal, chose parfaitement normal à une époque où la dialectologie est une pièce constitutive importante de l'appareil universitaire français.
} 
Mais l'approche ne manque pas de radicalité par certains aspects, dès lors que l'arabe paraît en effet infériorisé de fait dans ses fonctions. On lui donne un statut, mais dans des études qui n'ont pas grand-chose à voir avec le monde moderne, ce monde moderne associé au français qui exerce une fascination sur nombre de Tunisiens. Cela fonde bien-sûr un thème (constant par la suite) de l'opposition entre français (langue de l'actualité) et arabe (langue du passé). Cette langue n'est donc pas niée, mais claquemurée, ce qui probablement revient au même. La diglossie interne de l'arabe revient d'elle-même en négatif sur cette langue, surtout quand on constate ou qu'on estime que l'arabe dit « dialectal » ne s'écrit pas, ou que les formes plus ou moins classiques de l'enseignement coranique sont parfaitement anachroniques sociologiquement ${ }^{30}$. Tout cela, naturellement, engendra une frustration sensible, y compris au sein des élites francisées dans lesquelles allaient se recruter les cadres nationalistes de l'indépendance tunisienne.

C'est cet ensemble de principe qui fait que les élites instruites ou à instruire, n'eurent guère le choix au cours du protectorat, voyant bien, face à un tel système politique et sociolinguistique, où se trouvaient les leviers du pouvoir et les sources de maintien ou d'accroissement de ce pouvoir pour les familles de notables, pour réussir au mieux également. Le protectorat intégra donc ainsi une partie de la masse arabo-musulmane, prioritairement les classes sociales élevées, mais aussi plus généralement différentes sections de la population en contact direct avec la population européenne (travailleurs, employés divers etc.), avec les administrations (militaires, cadres subalternes des différents corps), ou quelque peu touchées par la scolarisation. On ne perdra pas de vue que la Tunisie, dès cette époque, fait figure de vedette en Afrique du Nord pour ce qui concerne l'éducation publique. Avec du recul, les plans de scolarisation paraissent ambitieux pour le plus petit des trois pays du Maghreb, efforts proportionnellement plus importants qu'au Maroc, et même qu'en Algérie (du moins pour la population arabo-musulmane $)^{31}$. Cet effort, tout en maintenant une forme de veille pour la connaissance de l'arabe (par le système franco-arabe notamment ${ }^{32}$ ) favorisa une certaine expansion du français dans des couches sociales qui par elles-mêmes auraient probablement eu peu de raisons de le recevoir.

Finalement les catégories qui furent peu ou pas du tout exposées à la francisation se trouvèrent localisées dans certains prolétariats urbains et surtout ruraux, le paysannat en règle générale et différentes zones excentrées géographiquement ou sociologiquement.

Il n'en reste pas moins qu'on est assuré de la présence d'un français endogène de Tunisie, entendons un français véhiculaire lui-même étagé depuis des formes minimales (celles de la $r^{33}{ }^{33}$ ) à des formes plus soutenues dans les élites sécrétées par le protectorat français. C'est l'existence de ce français endogène, d'autant plus admis et partagé qu'il venait de loin (cf. le Collège Sadiki), qui permit la politique tunisienne des années suivantes.

Un dernier mot s'impose, à propos du berbère. Celui-ci est assurément le grand perdant du protectorat, lequel s'articule autour d'une opposition français vs. arabe. Le berbère ne trouve

\footnotetext{
${ }^{30}$ D'où la tirade usuelle, rituelle, sur les écoles coraniques (voir plus haut).

${ }^{31}$ Lanly (1970), suivant Albertini (1955), confirme une scolarisation supérieure des enfants musulmans en Tunisie, remarque se trouvant dans la lignée des faits observés plus hauts par Foncin (1900). Au début du XXI siècle, la Tunisie fait toujours figure de vedette dans le monde arabo-musulman pour ce qui concerne l'alphabétisation et la scolarisation publique.

${ }^{32} \mathrm{Au}$ sein duquel, il faut néanmoins le rappeler, l'arabe fut minoritaire quantitativement. On rappellera aussi que si le colonisateur favorisa un enseignement primaire de ce genre, les formations supérieures, universitaires, administratives, restèrent le plus souvent inaccessibles à la population arabo-musulmane (Sraieb, 2000).

${ }^{33}$ C'est en effet le français d'Afrique du Nord, l'une des variétés les plus célèbres en est le français « pied-noir » ou français régional (Lanly, 1970) dont nombre de caractéristiques typologiques se retrouvent dans le français parlé en Tunisie. On rappellera que ce français populaire commun fondait aussi son originalité sur la récupération d'interlectes méditerranéens antérieurs au protectorat, le sabir notamment.
} 
pas sa place dans cette opposition et apparaît déjà comme une langue résiduelle, privée de support écrit et culturel, une vraie langue de paysans excentrés (généralement au sud) bien qu'au début du protectorat les Français durent probablement rencontrer des groupes berbérophones dans la «dorsale» ou Atlas tunisien. Assurément, le berbère est traité autrement qu'en Algérie et surtout que dans le protectorat marocain où il constitua, en totale opposition avec la Tunisie, une pièce essentielle du dispositif. Cette cécité apparente du protectorat (on voit passer très peu de renseignements dans les encyclopédies et travaux divers de l'époque) trouve sa source bien sûr :

a) dans la faiblesse numérique des berbérophones (dès cette époque),

b) par contrecoup dans le faible intérêt des Français pour une langue non opposable à l'arabe dans une perspective politique ou d'aménagement linguistique (contrairement au Maroc et à l'Algérie),

c) dans la plus grande homogénéité et dynamique de l'arabe tunisien, que le protectorat allait indirectement soutenir par son système d'enseignement franco-arabe, complétant ainsi le système traditionnel des écoles coraniques et donnant définitivement à l'arabe un statut quasi-administratif et officiel. La résultante fut très normalement une minimisation statutaire accrue et par suite un recul accéléré du nombre de locuteurs de cette langue.

Lorsqu'en 1955 parut l'ouvrage de synthèse Initiation à la Tunisie (Despois, 1955), deux spécialistes émérites, de l'arabe (William Marçais) et du berbère (André Basset), donnèrent chacun un article sur l'arabe ou le berbère en Tunisie. Ces articles consacrent clairement la domination géographique et sociologique de l'arabe. Le travail d'André Basset notamment, basé alors sur des données antérieures à la deuxième guerre mondiale révèle, dès cette époque un recul important du berbère, déjà proche de ses positions géographiques actuelles.

\section{La Tunisie indépendante}

La Tunisie indépendante et les années qui précèdent cette indépendance sont profondément marquées par le rôle d'un homme politique en particulier, Habib Bourguiba. Il faut bien voir que les directions qui vont se dessiner en matière de positionnement politique, culturel et langagier sous la conduite de cet homme politique se trouvaient en réalité dans la logique profonde de ce qui avait précédé, outre la personnalité et la stature de l'homme. On peut l'écrire d'une autre manière : la gestion «bourguibienne », ou «tunisienne », des langues et identités n'aurait sans doute pas pu être transposée telle quelle ni en Algérie, ni au Maroc, toutes proportions gardées. Ce qui doit nous rappeler, humblement, que la concrétisation politique en actes (politique linguistique et culturelle) se trouve placée sous la contrainte puissante des conditionnements historiques, géographiques et identitaires. Des conditionnements, un passé, des dynamiques largement différents en Tunisie, au Maroc et en Algérie, à plus forte raison entièrement différents d'autres régions francophones en Afrique subsaharienne, en Amérique, ou en Asie.

Dans un discours de Habib Bourguiba au Québec (à la fin des années 60) ${ }^{34}$ on trouve à peu près tous les ingrédients dynamiques de la francophonie tunisienne, et, par extension peutêtre, ceux de la francophonie maghrébine.

1. La langue française est pour vous, hommes et femmes du Québec une partie de vous-mêmes dont vous avez su, et à quel prix, assurer la pérennité. A nous, il semblerait qu'elle soit venue du

\footnotetext{
${ }^{34}$ Le Président Bourguiba au Canada, extraits des discours d'Ottawa et de Montréal. Libre coopération, «La francophonie» (t. 2). Publié par l'Association Tunisienne Les amis de la France, 1968.
} 
colonisateur. Et pourtant, il ne nous semble pas que ce soit à lui en tant que tel, que nous la devons. Il est même de mon devoir d'évoquer, à ce propos, le souvenir de notre grand premier ministre Khéreddine. C'est lui, en effet, dont le turc était la langue maternelle, qui, le premier, a ouvert à des jeunes de chez nous le chemin des universités de France; c'est lui qui, le premier a introduit le français dans l'établissement secondaire qu'il a créé, ce Collège Sadiki auquel tant de mes compagnons de lutte comme moi-même, après bien d'autres devanciers, sont en grande partie redevables de ce qu'ils sont devenus et de ce qu'ils ont accompli pour le salut politique et pour le devenir de la Tunisie [.] Je peux bien dire que, dès cette époque et en dépit du jeu d'influences diverses qui s'exerçaient alors sur notre pays ${ }^{35}$, l'intelligentsia tunisienne avait déjà opté pour la langue française et pour une culture ouverte sur le monde moderne.

2. Vous dirai-je que votre exemple m'apparaît comme portant une leçon communicable aux hommes du continent auquel appartient la Tunisie ${ }^{36}$ ? La francophonie, je vous l'ai dit, n'est pas pour nous un antique héritage. C'est une greffe. Il a suffi de quelques générations pour que, malgré les intempéries, elle prenne, se développe, donne naissance à un arbre vigoureux. Les Africains ont toutes raisons de méditer votre exemple et d'apprendre de vous quels soins opiniâtres il faut lui apporter.

3. Que disais-je alors" 37 ? Ceci : «Je n'aime pas beaucoup le mot "francophonie" mais, enfin je dois reconnaître que la "francophonie" représente en Afrique une réalité. Non seulement parce qu'elle met en contact privilégié les pays où le français est langue officielle et ceux où elle est langue de travail, mais parce qu'elle rend les uns et les autres participants à un même univers culturel, parce qu'elle rend les uns et les autres plus à même de découvrir, même au-delà de la langue, ce qui les unit. C'est donc une sorte de Commonwealth que je voudrais voir s'établir entre eux, une communauté qui respecte les souverainetés de chacun et harmonise les efforts de tous ».

Les discours du Président Bourguiba mériteraient un examen plus fin, dans un exercice qui devrait réunir les ressources de la sociolinguistique, de l'histoire et de la pragmatique, de l'analyse politique bien évidemment ${ }^{38}$. À défaut de pouvoir faire cela franchement dans ce cadre, je me bornerai à quelques remarques afin de définir les contours essentiels et l'environnement de la francophonie tunisienne durant la seconde moitié du $\mathrm{XX}^{\mathrm{e}}$ siècle.

La première chose qui frappe, bien qu'on parle ici expressément «d'héritage », est la difficulté à assumer la francophonie comme composante normale de l'identité tunisienne. Mais même cela, dans la logique plus haut soulignée, doit se faire sans passion apparente, du moins sans choc majeur.

Si la langue française est pour des Canadiens « une partie d'eux-mêmes », un cortège de formulations montre bien que tel ne peut être le cas pour une Tunisie posée ici politiquement comme ambassadrice du Maghreb et plus largement de l'Afrique. Le point qui fait mal, ou qui est présumé faire mal ${ }^{39}$, est très exactement celui de l'imposition, de la « greffe », un thème qu'il faut donc évacuer par différentes stratégies, et que l'Union française (voir plus bas) ne pouvait au contraire qu'aviver. Mais toutes ces stratégies me semblent déjà devoir être mises en relation avec ce relatif manque d'agressivité souligné pour la Tunisie, ou de politesse linguistique si l'on préfère. En outre la greffe n'exclut pas le travail (d'où le terme de «soins opiniâtres », extrait 2), ce qui d'une manière ou d'une autre nous ramène toujours au caractère non naturel de l'implantation. Bien évidemment, s'il fallait vraiment commenter cela, force

\footnotetext{
${ }^{35}$ Voir plus haut par exemple les précédents de l'influence italienne, qui, par proximité, continuaient de jouer à cette époque. Mais cette époque est surtout celle du panarabisme nassérien, il y a là une source supplémentaire de difficulté (du coup preuve du talent du Président Bourguiba) pour assumer directement le français dans un tel contexte.

${ }^{36}$ Il vient de souligner les combats, la volonté, la vitalité et l'action des Québécois pour préserver et développer le «tronc français» auquel ils se rattachent directement.

${ }^{37}$ Il évoque ici un autre discours, du 24 novembre 1965, à l'Université de Dakar.

${ }^{38}$ Cette approche politique lato-sensu est faite notamment dans un ouvrage récent (Camau \& Geisser, 2004).

${ }^{39}$ Car il s'agit bien d'un discours politique et diplomatique qui, au-delà de la personne qui prononce le discours, configure en principe la posture d'un État et celle d'un peuple. Une certaine mise en scène dans le cadre de propos «politiquement corrects » (comme on dirait aujourd'hui), ne peut donc être exclue.
} 
serait de dire qu'en matière de langage rien n'est proprement naturel, tout est affaire de contacts, de rapports de force, d'interdépendance, de compétition. Enfin, on ne peut laisser de côté les « intempéries », référence indirecte aux difficultés pour assumer la langue française, les chocs directs ou indirects avec l'identité arabe, ou arabo-musulmane, tous les risques de rejets de la greffe donc.

La remontée au Collège Sadiki est une de ces stratégies qui permettent d'envisager positivement la langue française tout en gardant une distance suffisante. Cet établissement, effectivement très important pour la formation des élites socio-politiques tunisiennes de l'indépendance, serait historiquement la véritable agence d'introduction du français dans les élites (paragraphe 1). Cette attitude correspond à une réalité déjà entrevue plus haut, et à l'histoire personnelle du Président Bourguiba ${ }^{40}$. Mais il faut souligner qu'elle permet de développer en creux toute une série de caractérisations fondamentales, importantes pour nous. L'une d'entre elles serait donc que le français fut bien d'origine élitaire, ce que l'on a remarqué avant et pendant le protectorat. L'espace d'affirmation et de confirmation de cette langue serait depuis le début et par la suite celui des élites tunisiennes, élites sociales, élites politiques, même si des contradictions apparurent avec le temps entre ces deux types d'élites ${ }^{41}$. Les élites sociales tunisiennes ont en effet constitué tout au long du XXe siècle un emplacement sociolinguistique normal de pratique de la langue française, non seulement à titre de langue de pouvoir mais plus spontanément et naturellement aussi comme l'une des langues usuelles et fonctionnelles de la famille et du groupe socio-politique. Il est très frappant par exemple de constater que différentes enquêtes dans les années 1960-1970 et par la suite révélaient l'existence d'une francophonie autochtone déclarée et plutôt assumée, mieux assumée d'ailleurs qu'en Algérie ou dans l'autre protectorat par exemple ${ }^{42}$. Sous nos yeux encore, le français est largement resté une langue de reconnaissance, au moins partielle, des élites cultivées. Une particularité tunisienne très marquée doit être soulignée, celle des mariages « mixtes » qui jusqu'à nos jours tient une place importante dans la société tunisienne et soustend largement un enracinement local de la langue française. Bien entendu, le discours officiel d'Habib Bourguiba ne pouvait s'appuyer politiquement sur ce genre de considération. Ce n'est pas une telle francophonie locale, endogène, qu'il veut évoquer, bien que lui-même s'y rattache personnellement. C'est bien d'une francophonie exogène qu'il nous parle, du français comme langue d'ouverture sur la modernité, donnant un contour palpable et acceptable à un axe qui traversera par la suite l'ensemble des opinions et des politiques éducatives maghrébines. C'est celui de l'identification du français comme langue non autochtone, étrangère, mais aussi comme langue indispensable d'ouverture sur le monde. Mais en même temps on sent bien l'impossibilité structurelle, idéologique et politique, de tenir un quelconque discours qui légitimerait un français endogène, donc une appropriation de la langue française qui existe pourtant dans les faits.

\footnotetext{
${ }^{40}$ Beaucoup d'autres soulignent cela. Par exemple, lors d'un entretien avec Béji Caïd Essebsi, « Sadiki, dont j'ai obtenu le diplôme, était l'école dans le sens large du terme, une école de patriotisme et de culture. Nous les Sadikiens, nous avons tous été bilingues, de culture arabe et de culture française. » (Camau \& Geisser, 2004, p. 578).

${ }^{41}$ Après l'indépendance de la Tunisie, la réorientation vers le patrimoine arabe (arabisation), combinée aux efforts/difficultés pour maintenir une présence légitimée de la langue française, provoqua dans l'ensemble des pays du Maghreb, en Tunisie donc aussi, des alternances de phases tantôt favorables à l'arabisation (système scolaire, administration etc.), tantôt favorables au contraire à un retour accru du français dans les formations. Ce système pendulaire est vu et a toujours été vu comme mauvais par l'ensemble des partenaires, tendant à créer constamment des distorsions entre les Tunisiens suivant qu'ils ont été formés à telle époque ou à telle autre. C'est là un système d'accordéon qui relance constamment les processus d'affrontement et de méfiance.

${ }^{42}$ On dispose de statistiques de 1958 pour l'Algérie, 1960 pour la Tunisie, 1969 pour le Maroc. Il ne peut être question d'entrer ici dans le détail, mais il y aurait à l'époque 180.000 locuteurs déclarés de français en Tunisie, contre 106.000 au Maroc et 78.000 en Algérie. S'ils sont fondés, les chiffres parlent d'eux-mêmes. Pour ces statistiques, voir W.-F. Mackey (1976, 462-463).
} 
On comprend alors que l'idée d'un Commonwealth à la française ait pu devenir dans ce cadre une solution pragmatique et politique (extrait 3), surtout à une époque où l'on venait à peine de sortir de ce qui s'appelait quelques années auparavant l'Union française. Celle-ci, avait été créée par la constitution française de 1946, et modifiée en Communauté française par la constitution de la $\mathrm{V}^{\mathrm{e}}$ République, en 1958. De tels dispositifs, mis en place au moment où des États (comme la Tunisie ou le Maroc) étaient déjà indépendants et d'autres aspiraient à le devenir (Afrique Occidentale Française, Afrique Équatoriale Française, Algérie), apparurent assez clairement comme des formes de prolongement ou de survie de l'Empire colonial français. Pour mieux juger de la distance prise par Bourguiba et du climat d'une époque, on peut citer un texte comme celui-ci :

L'ancienne conception « Métropole-Colonies » semble abolie. L'expression Empire français est périmée. Nous avons d'ailleurs un Ministère de la France d'Outre-mer, qui en est la désignation officielle. L'expression Union française est adoptée depuis 1945, dans le sens précis de «Fédération », impliquant le respect des patries indigènes. Ainsi se trouve supprimé, du moins chez nous, le «pacte colonial» en vigueur jusqu'à la dernière guerre. Comment s'organiseront les rapports de la mère-patrie et des anciennes colonies, devenus pays libres et d'administration autonome? ${ }^{43}$

Il faut évidemment tenir compte de ce genre de présentation pour comprendre à quel point assumer le fameux « héritage français » pouvait être problématique au sud de la Méditerranée. Dire que le français est associé aux élites, c'est donc faire pointer l'idée, dès Bourguiba, qu'il n'avait et n'aurait guère de chance d'adhérer à la masse, «arabo-berbère » ou "arabomusulmane », sauf à admettre une diffusion démocratique réussie de la langue française comme langue de progrès et par contrecoup la caractérisation du bloc identitaire arabomusulman comme un socle d'inertie passéiste. Peu importe au fond comment l'on se positionne politiquement par rapport à cette dualité symbolique, force est de voir qu'à peu près tout le monde la partage et qu'elle est devenue un véritable standard de cadrage du monde maghrébin. Les plus hostiles à la francophonie ou à une forme d'acculturation européenne y verront un ensemble de raisons fondamentales pour réorienter le Maghreb sur ce que l'on considère être sa véritable trame identitaire, celle dont il se serait trop éloigné depuis la colonisation française notamment : l'arabe, l'arabité, l'islam. Mais dans le même temps, à des degrés divers, l'ensemble des progressistes ou modernistes verront se fonder une double contrainte pour la Tunisie notamment (et le Maghreb) :

a) maintenir un haut niveau de francophonie à même d'arrimer le pays à une forme d'internationalité et de performativité socio-économique (point de vue $\mathrm{du}$ développement collectif) tout en permettant aux individus d'être mis en contact avec des valeurs de démocratie, de liberté, de droit etc. (point de vue du développement individuel). Car en effet les défenseurs Tunisiens de la francophonie tunisienne mettent d'eux-mêmes en avant ces différents aspects.

b) rendre l'arabe (et l'arabisation du système sociolinguistique général) capables de procurer aux Tunisiens un accès aux mêmes valeurs et technologies, internationalité et développement individuel donc. Le problème réside alors dans l'aptitude ou l'inaptitude déclarées ou présumées de l'arabe à prendre en charge des secteurs sociaux et culturels de ce genre.

Le panachage entre ce (a) et ce (b) est en théorie possible, et il se décline à travers différentes moutures de bilinguisme, dont celle que propose l'État dans ses lois.

\footnotetext{
${ }^{43}$ Nouvelle encyclopédie didactique Quillet, tome II, page 534 (« Les Pays d'Outre-mer de l'Union Française »).
} 
Mais on voit bien qu'en dépit des progrès officiels récents de l'arabisation, les problèmes n'ont guère changé de nature depuis un demi-siècle, voire même depuis le début du $\mathrm{XX}^{\mathrm{e}}$ siècle. Un propos illustrant ce genre de difficultés est cité dans le site de Jacques Leclerc ${ }^{44}$. Il pourrait paraître isolé, mais lorsqu'on a travaillé et enquêté en Tunisie on sait bien qu'il ne l'est pas et qu'il est représentatif de réalités qui se retrouvent aussi dans le reste du Maghreb.

Les efforts entrepris depuis l'indépendance pour introduire l'arabe dans certains secteurs de l'administration jusque-là entièrement francisés sont restés à peu près sans effet. Tel est le cas, par exemple, de l'administration des Postes et Télécommunications : les divers imprimés mis à la disposition du public, dans les guichets, sont libellés dans les deux langues. Mais une infime proportion (1 à $2 \%)$ des utilisateurs s'intéresse à la version arabe de ces imprimés. Quant au personnel, il ne lui viendrait jamais à l'idée d'utiliser cette version [... $]^{45}$

\section{Systémique du paysage linguistique de la Tunisie, éléments du puzzle et prospective}

Le propos précédent, pour être représentatif, n'en est pas moins insuffisant. Il faut en tout cas le compléter.

L'arabisation a considérablement progressé dans les faits depuis une vingtaine d'années. Comme nous l'avons remarqué plus haut, des facteurs systémiques ont, de longue date, favorisé cette progression, une fois encore moins tendue qu'elle ne l'apparaît plus à l'ouest.

Parmi ces facteurs favorables, on doit encore rappeler que l'homogénéité de l'arabe tunisien est grande. Pour comparer avec le Maroc par exemple, on aurait du mal à trouver des soussystèmes dialectaux aussi tranchés que le sont le «marocain » oriental et le «marocain » occidental, sans compter les variantes régionales de l'arabe parlé par des Berbères eux-mêmes très divers, du Rif au Sous, en passant par le Moyen et le Haut-Atlas.

Il y a aussi la place désormais infime du berbère, qui semble légitimer la mécanique d'arabisation, alors qu'au Maroc ou en Algérie le berbère posera toujours autant de problèmes à l'État tant qu'il disposera d'un vivier confortable. C'est bien pourquoi j'ai insisté sur le fait que cette langue n'est même plus «vue » en Tunisie dans la plupart des cas. Quel que soit son intérêt intrinsèque, son intérêt écolinguistique notamment, elle constitue désormais la portion négligeable du système en termes de poids, de mentalités et de dynamiques.

$\mathrm{Au}$ faible fractionnement dialectal s'ajoute encore un autre facteur qu'on évoque assez peu. C'est celui de la position géographique de la Tunisie dans le monde arabophone. Elle se trouve, avec la Libye (très voisine sur un plan géolinguistique) dans une position de pivot entre les deux macro-zones conventionnellement dénommées Machrek, ou Machreq (arabe « oriental ») et Maghreb (arabe « occidental »). Que l'arabe tunisien fasse plutôt partie par ses traits du bloc maghrébin n'est guère contestable ${ }^{46}$. Mais en revanche la position géographique, l'homogénéité soulignée, «l'absence» de berbère, permettent certainement aux opinions publiques d'accueillir avec moins de réserves les discours sur l'arabité ou l'arabo-islamité, régulièrement venus du Moyen-Orient et associés à la langue arabe moyen-orientale, à des époques différentes, et sous nos yeux encore.

Cet ensemble de considérations explique pour l'essentiel pourquoi l'arabisation a fortement progressé en Tunisie depuis une vingtaine d'années. Comment cette arabisation régulière de l'État et de ses structures affectera-t-elle les contours de la francophonie tunisienne, c'est un dossier trop large et prospectif qu'on ne peut évoquer dans ce cadre restreint.

\footnotetext{
${ }^{44}$ http://www.tlfq.ulaval.ca/axl/afrique/tunisie.htm

${ }^{45}$ Le site précise que le propos émane d'un « universitaire tunisien ».

${ }^{46}$ Pour une approche géo-typologique, voir Philippe Marçais (1977). Depuis quelques années, un projet d'atlas linguistique de la Tunisie se développe. Voir par exemple Taïeb Baccouche \& Salah Mejri (2004).
} 
Néanmoins, pour s'en tenir aux faits, la place de l'arabe n'a cessé d'augmenter ces dernières années :

- dans les ministères et administrations de l'État. Si au début de l'indépendance la disparité était grande dans les volumes attribués à l'arabe et au français (lequel était largement privilégié), toutes les administrations communiquent désormais en arabe, même si des difficultés se font jour parfois et semblent imposer certaines mesures coercitives. On peut donc parler d'une inversion historique, globalement confortée, étalonnée par les médias (télévision tunisienne publique principalement arabophone)

- dans le domaine de l'enseignement, où l'arabe, à l'origine minoritaire, a augmenté sa masse horaire tout en investissant des zones d'enseignement où il n'intervenait pas ou peu à la sortie du protectorat (par exemple les sciences). Différents problèmes se posent encore, notamment dans les enseignements supérieurs ${ }^{47}$, mais l'arabisation se poursuit toujours, à un rythme élevé semble-t-il, d'autant que les cadres intermédiaires et techniques ont été eux-mêmes de plus en plus formés dans un système principalement arabophone.

Cette représentation s'est encore et surtout affirmée dans la symbolique et les statuts. Ainsi la constitution de la république tunisienne de 1992, modifiée en 2002, stipule que "La Tunisie est un État libre, indépendant, souverain ; sa religion est l'Islam, sa langue l'arabe et son régime, la République." L'arabe est donc bien langue officielle constitutionnelle de la Tunisie, en même temps que l'islam lui est associé plus étroitement que jamais.

Cette modification de la constitution rend possible l'évacuation définitive du français sur les terrains officiels de l'État, mais surtout la conversion définitive de cette langue en langue d'ouverture, internationale, c'est-à-dire pour finir de langue non intrinsèquement constitutive de l'identité profonde tunisienne, ce qui est indirectement le cas aussi de toute autre composante cultuelle autre que l'islam. La république est arabophone et musulmane.

Cette systématique transparaît désormais de manière rituelle et répétitive dans de nombreux textes officiels. Ainsi une loi de 1993 prévoit-elle logiquement que les lois, décrets et arrêtés divers paraissent au Journal Officiel de la République Tunisienne en langue arabe. Un alinéa prévoit ensuite : "Ils sont publiés également en une autre langue, et ce, uniquement à titre d'information." 48

Une loi d'orientation de l'enseignement (2002) permet de mesurer en même temps la progression de l'arabisation, de l'affirmation arabo-islamique et de la spécialisation systémique du français, indirectement affichée, puisque le français n'est pas ou plus mentionné.

L'article 3 précise :

L'éducation a pour finalité d'élever les élèves dans la fidélité à la Tunisie et la loyauté à son égard, ainsi que dans l'amour de la patrie et la fierté de lui appartenir. Elle affermit en eux la conscience de l'identité nationale et le sentiment d'appartenance à une civilisation aux dimensions nationale, maghrébine, arabe, islamique africaine et méditerranéenne, en même temps qu'elle renforce l'ouverture sur la civilisation universelle.

\footnotetext{
${ }^{47}$ Dont on se souvient qu'ils étaient à peu près intégralement francophones à la fin du protectorat. On touche un problème délicat pour l'ensemble des pays du Maghreb : dans quelle mesure faut-il arabiser l'enseignement supérieur et la recherche ? Quels seraient l'intérêt et l'incidence économique d'une telle démarche dans un monde où des langues internationales autres que l'arabe sont requises ?

48 On peut légitimement se demander dans quelle langue et pour l'information de qui. Des pays étrangers (européens) ? Des Tunisiens francophones ou imparfaitement arabophones?
} 


\section{L'article 51:}

La langue arabe est enseignée dans tous les cycles de l'enseignement de façon à garantir sa maîtrise à la fois comme moyen de communication et de culture, et son utilisation pour apprendre et pour produire dans les différents domaines du savoir.

Les langues étrangères sont enseignées dès le premier cycle de l'enseignement en tant qu'outils de communication et moyens d'accès direct aux productions de la pensée universelle et ce qu'elle véhicule comme techniques, théories scientifiques et valeurs civilisationelles, afin de préparer les jeunes à suivre leur évolution et à y contribuer d'une manière qui permette à la fois d'enrichir la culture nationale et d'assurer son interaction avec la culture universelle. ${ }^{49}$

Ces textes sont suffisamment explicites ${ }^{50}$. Le plus frappant sans doute est que le français, pour important qu'il puisse être jugé dans les faits et sur le terrain (c'est-à-dire les pratiques effectives, les réalités quotidiennes de la Tunisie), est désormais radicalement fondu dans un collectif «langues étrangères». On ne le nomme même plus, ce qui élimine aussi tout caractère éventuel de langue étrangère préférentielle, mais ce qui en même temps ne trompe personne.

Cela sonne-t-il le glas de la langue française en Tunisie ? Probablement pas, ou en apparence seulement : les textes officiels, en dépit de leurs ambitions et de leur légitimité, ne peuvent modifier radicalement les paysages sociolinguistiques car ils n'ont pas de prise directe sur les déterminations socio-historiques qui fondent ces paysages. En revanche ils peuvent certainement provoquer des avancées en trompe-l'œil, ou préparer les conditions de changements ultérieurs. L'un des paradoxes de la Tunisie et de l'ensemble du Maghreb est que l'arabisation progresse partout, car le Maghreb doit construire son avenir avec le monde arabe, c'est indéniable. Mais dans le même temps se renforce l'idée que cet avenir est économiquement et culturellement contraint par un système de relations avec l'Europe, plus proche et incontournable que jamais, bien que les détroits maritimes n'aient pas rétréci depuis le siècle dernier.

Si une réorientation et un ancrage européens s'annoncent plus pour des raisons économiques que parce que l'État tunisien le souhaite dans les textes que nous avons vu (avec retours variables de l'anglais, de l'italien, de l'espagnol suivant les parties du Maghreb considérées), il est également probable que le français, conservera mécaniquement son rôle de "passeur » langagier et culturel et pourra ainsi renforcer certains de ses rôles. À cet égard, la Tunisie et le Maroc, l'Algérie dans une moindre mesure, ne peuvent s'abstraire d'une proximité historique et géographique constante de la Romania, et pour interagir avec la Romania il faut prioritairement des langues romanes véhiculaires. Cette Romania est là depuis très longtemps, c'est un partenaire immédiat. Ainsi que je l'ai écrit à plusieurs reprises, la dimension romane est en réalité inscrite géolinguistiquement et géoculturellement dans le tissu maghrébin ${ }^{51}$. On a probablement tort de réduire cette présence (celle du français notamment) aux manœuvres coloniales, car celles-ci pour frustrantes et injustes qu'elles aient pu être, concrétisent une relation d'ensemble beaucoup plus ancienne, permanente, et qui certainement ne risque pas de s'évaporer en quelques décennies. Une relation nécessaire, constitutive, consubstantielle, qu'aucun texte ne pourra probablement évacuer, pas plus que la puissance coloniale française n'avait réussi en son temps à détacher la Tunisie et le Maghreb de son ancrage araboislamique, pour les mêmes raisons.

49 Loi d'orientation $\mathrm{n}^{\circ} 2002-80$ du 23 juillet 2002, disponible en français sur le lien : http://www.edunet.tn/ressources/reforme/orientationfr.pdf

50 Soulignons aussi que l'examen des manuels scolaires tunisiens à travers le temps confirme certaines orientations culturelles ayant conduit à ces divers lois ou décrets. Ce point ne peut être développé ici. Un ouvrage récent procurera des éclairages intéressants dans cette optique (Abbassi, 2009).

${ }^{51}$ Voir par exemple Manzano (2004) et plus généralement les sources indiquées plus bas. 
Pour que le français disparaisse de lui-même du paysage tunisien, il faudrait que les conditions qui l'ont amené ici et qui ont justifié son maintien comme langue d'usage en Tunisie disparaissent, et que d'autres langues étrangères puissent se substituer à lui en reprenant l'ensemble des fonctions qu'il assure ou assurait. Sans compter la densité concrète, identitaire et culturelle, de l'attachement à cette langue et à cette culture.

On voit bien que tel n'est pas le cas. Les langues européennes, qu'elles soient romanes ou germaniques $^{52}$, ne sont pas égales ou jugées égales au français, car elles ne peuvent dire la même chose dans la bouche, dans l'esprit, d'un Maghrébin.

J'ai écrit plus haut que le français jouait le rôle de "passeur », passeur d'idées, passeur de conceptions du monde. Beaucoup d'intellectuels tunisiens expriment cette idée, d'une manière ou d'une autre, c'est un véritable leitmotiv. Le français est pour nombre de locuteurs actuels la langue qui permet de s'extraire d'une forme d'enfermement arabo-islamique, culturel mais aussi économique, c'est là une constante de discours exprimée sous des formes plus ou moins atténuées. Cette échappée pourrait se concevoir avec d'autres langues, l'anglais et l'italien en particulier. Mais si de telles options, pour l'instant du moins, ne conviennent pas et ne convainquent pas, c'est bien parce qu'en un siècle et demi l'identité maghrébine s'est transformée en profondeur et que le français est sans doute devenu une langue du Maghreb ${ }^{53}$. Comme j'essaye de le faire comprendre depuis plusieurs années, l'identité maghrébine est en réalité une identité composite, d'interface, fondée notamment sur trois piliers ou pôles. On a parfaitement le droit d'ignorer cela sur un plan politique, pour favoriser une option ou une autre et c'est ce que l'Histoire du Maghreb, colonial ou indépendant a clairement prouvé. Chacun peut alors nier ces réalités profondes à sa manière : discours colonial, discours francophonique tantôt passéiste ${ }^{54}$ tantôt plus modernisé, discours arabo-islamiste ${ }^{55}$, discours berbériste militant etc. Tout le monde a raison et tout le monde a tort, car en fin de compte tous ces discours se brisent sur le socle maghrébin, fondamentalement ternaire comme cela est rappelé au début de la contribution.

À cet égard, il y a pourtant une originalité tunisienne : le pôle berbère y est désormais presque éteint, ce qui éloigne nettement de l'Algérie et du Maroc. On passerait donc en ce moment d'un système tripolaire à un système binaire ou bipolaire, plus proche de ce que l'on connaît en général dans les pays européens voisins comme l'Italie, la France ou même l'Espagne. Dans ces trois pays, en dépit de remontées relativement récentes des langues dites « régionales », on ne peut ignorer que les langues des États ont progressé à une vitesse fulgurante au cours du $\mathrm{XX}^{\mathrm{e}}$ siècle, car elles se trouvaient constamment opposées à des langues locales particulières qui ne pouvaient leur offrir de résistance valable dans le cadre d'une généralisation des langues véhiculaires (Manzano, 2008). Logiquement, la binarité prochaine devrait donc profiter à l'arabe et à l'arabisation, appuyée sur les modifications de statut évoquées et sur le fait que de plus en plus de locuteurs sont formés, que de plus en plus d'espaces sont investis par l'arabe. Mais cette progression prévisible bute déjà et butera de plus en plus sur quelques éléments que nous considérerons pour finir :

\footnotetext{
52 On pense bien entendu à l'anglais, mais différentes autres langues germaniques exercent une pression sur la Tunisie, notamment par le canal du tourisme (Manzano, 1996).

${ }^{53}$ Ce type d'approche était bien posé dès les années 80 par Gilbert Grandguillaume (Grandguillaume, 1983), qui rappelait dans sa conclusion (p. 149), la position très particulière du français au Maghreb. Plus récemment, Samir Marzouki (Marzouki, 2007) a utilement prolongé cet axe de réflexion.

${ }^{54}$ C'était bien la chimère d'une langue française s'imposant et se répandant au Maghreb en raison de ses vertus présumées d'internationalité, de clarté et d'intellectualité, comme beaucoup le croyaient durant la première moitié du $\mathrm{XX}^{\mathrm{e}}$ siècle.

${ }^{55}$ Avec lui-même plusieurs variantes. De celle qui est en ce moment portée par l'État jusqu'aux formes les plus extrémistes de rejet des langues et cultures étrangères, du français en première ligne.
} 
- d'abord celui de l'internationalité et de l'ouverture. Tant que l'arabe ne pourra vraiment concurrencer le français, ou d'autres langues d'origine européenne, dans les domaines de l'économie, des savoirs et du monde contemporains (ce que l'on résume -un peu trop simplement toutefois- sous le concept de mondialisation), un espace plus ou moins large lui échappera constamment à l'échelle internationale mais aussi à l'intérieur des frontières des États maghrébins. Évidemment, cela profitera au français ou à d'autres langues «étrangères », ce qui sous-tend au fond les textes et articles récents plus haut mentionnés.

- d'autre part, le système tripolaire maghrébin classique tendant à devenir caduc en Tunisie, force est de voir qu'il est presque automatiquement remplacé par un nouveau modèle tripolaire qui, cette fois, pourrait mettre en relation de complémentarité et/ou de compétition :

(a) l'arabe « originel», mais en réalité d'origine non maghrébine (sous ses diverses appellations de classique, littéral etc.),

(b) l'arabe dialectal, à propension nationale et identitaire, celui-ci au contraire réellement maghrébin et à ce titre reprenant à son compte différents traits du pôle berbère antérieur,

(c) le français (ou, à terme, d'autres langues européennes concurrentes).

On reconnaît là des phénomènes récurrents depuis plusieurs années, mais qui s'imposeront avec de plus en plus d'acuité. Quoi qu'on fasse, le rapport (a) vs. (b) entraînera à nouveau dans l'opposition latente entre des normes endogènes et des normes exogènes sur fond identitaire ${ }^{56}$, le (c) pouvant devenir presque de facto un arbitre.

Enfin le développement de l'arabisation amènera à reposer régulièrement la question de la nature du français. Les textes officiels tendent et tendront encore à le présenter systématiquement comme une langue étrangère complémentaire. Mais cette qualification se heurtera pour un temps, difficile à apprécier, sur la production continue de normes endogènes de cette langue, constatées par les linguistes ${ }^{57}$, et significatives d'une perception plutôt positive, identitaire et régionale. Tant que de telles normes et de telles pratiques subsisteront, tant que se maintiendront aussi les phénomènes si importants de code-switching et toutes sortes d'interférences (preuves d'une véritable alternance et d'un contact des langues sur place $)^{58}$, tant qu'une partie des élites au moins continuera d'assumer cette langue, dans la mesure aussi où les relations avec l'Europe ne feront que se préciser, on disposera d'un faisceau permettant au français de rester une langue du terrain tunisien.

\footnotetext{
${ }^{56}$ C'est un problème souvent abordé en Tunisie sous un angle négatif et que Mohammed Benrabah (1994) avait évoqué pour l'Algérie notamment, sous la dénomination de « haine de soi ».

57 Naffati-Queffélec (2004) envisagent dans un chapitre particulier une brève caractérisation typologique de telles normes ( 8 «Les variétés du français », 85-94).

${ }^{58}$ Les études sur les interférences, les contacts entre le français et l'arabe, ont été nombreuses. On peut citer parmi d'autres celles de Foued Laroussi (1991, 1999). Antérieurement, dans les années 1970, Juliette GarmadiLe Cloirec avait développé des observations sur le français parlé et écrit en Tunisie $(1970,1977)$ et l'on pense aussi aux travaux pionniers du CERES (depuis 1965) et de la Revue tunisienne des sciences sociales animé par des chercheurs comme Salah Garmadi ou Taïeb Baccouche.
} 


\section{Éléments de bibliographie}

Abbassi, Driss. 2009. Quand la Tunisie s'invente: entre Orient et Occident, des imaginaires politiques. Paris: Autrement. 157 pages.

Albertini, Eugène (dir.). 1955. L'Afrique du Nord française dans l'histoire. Lyon : Archat. 375 pages.

Baccouche, Taïeb \& Mejri, Salah. 2004. L'Atlas linguistique de Tunisie : les questionnaires. Tunis, Paris : Ambassade de France en Tunisie, Maisonneuve et Larose. 103 pages.

Benrabah, Mohamed. 1994. "L'arabe algérien, véhicule de la modernité ». Cahiers de Linguistique Sociale 22 (Laroussi, dir.). 33-43.

Camau, Michel \& Geisser, Vincent (dir.). 2004. Habib Bourguiba : la trace et l'héritage. Paris : Karthala. 663 pages.

Despois, Jean (dir.) 1950. Initiation à la Tunisie. Paris : Maisonneuve. 397 pages.

Dubois Colette, Kasabarian Jean-Michel \& Queffélec Ambroise (éd.). 2000. L'expansion du français dans les Suds (XVe-XXe siècles). Aix-en-Provence : Publications de l'Université de Provence. 348 pages.

Foncin, Pierre. 1900. La langue française dans le monde. Paris : Alliance Française. 299 pages.

Garmadi-Le Cloirec, Juliette. 1970. «L'interférence grammaticale ». Revue tunisienne des sciences sociales, 22. 203-218.

Garmadi-Le Cloirec, Juliette. 1977. «Remarques sur la syntaxe du français de Tunisie». Langue française 35. 86-91.

Grandguillaume, Gilbert. 1983. Arabisation et politique linguistique au Maghreb. Paris : Maisonneuve et Larose. 214 pages.

Guernier, Eugène (dir.). 1942. L'Encyclopédie coloniale et maritime [ECM]. Volume La Tunisie. Paris : Encyclopédie coloniale et maritime. 447 pages.

Hagège, Claude \& Zarca, Bernard. 2001. Les Juifs et la France en Tunisie. Les bénéfices d'une relation triangulaire. Le Mouvement Social 2001/4 ( $\left.\mathrm{n}^{\circ} 197\right)$. 9-28.

Lanier, Léon. 1889. L'Afrique, choix de lectures géographiques. Paris : Belin. 920 pages.

Lanly, Albert. 1970. Le français d'Afrique du Nord, étude linguistique. Paris : Bordas. 367 pages.

Laroussi, Foued. 1991. L'alternance du code arabe dialectal/français. Etude de quelques situations dans la ville de Sfax (Tunisie). Rouen: Thèse de doctorat, Université de Rouen. 402 pages. 
Laroussi, Foued (dir.). 1993. Minoration linguistique au Maghreb. [Numéro spécial]. Cahiers de linguistique sociale 22. 125 pages.

Laroussi, Foued. 1999. « Un nouveau regard sur la situation linguistique tunisienne ». Cahiers de sociolinguistique 4. 155-169.

Mackey, William-Francis. 1976. Bilinguisme et contact des langues. Paris : Klincksieck. 534 pages.

Manzano, Francis. 1993. «Des Maghrébins lettrés observent le paysage linguistique d'Afrique du Nord ». Cahiers de Linguistique Sociale, 22 (Laroussi, dir.). 91-107.

Manzano, Francis. 1996. «Sur les mécanismes du paysage sociolinguistique et identitaire d'Afrique du Nord ». Langage et Société n ${ }^{\circ}$ 75. 5-44.

https://www.researchgate.net/profile/Francis_Manzano

https://hal-univ-lyon3.archives-ouvertes.fr/hal-00347698

Manzano, Francis \& Krier, Fernande. 1999. Langues du Maghreb et du sud méditerranéen. [Numéro spécial]. Cahiers de sociolinguistique $\mathrm{n}^{\circ} 4$, Presses Universitaires de Rennes. 170 pages.

Manzano, Francis. 2004. « À propos d'un corpus de sociolinguistique maghrébine ». Compterendu de Naffati Habiba \& Queffélec Ambroise, Le français en Tunisie, [Numéro spécial], Le Français en Afrique $n^{\circ} 18$. GLOTTOPOL Revue de sociolinguistique en ligne 4.

http://glottopol.univ-rouen.fr/telecharger/comptesrendus/crmanzano.htm

Manzano, Francis. 2007. «Sur l'implantation du français au Maghreb: systémique et fractures identitaires au tournant des $\mathrm{XIX}^{\mathrm{e}}$ et $\mathrm{XX}^{\mathrm{e}}$ siècles ». Le français en Afrique $\mathrm{n}^{\circ}$ 22. 5-42.

https://www.researchgate.net/profile/Francis_Manzano

https://hal-univ-lyon3.archives-ouvertes.fr/hal-00357266

Manzano, Francis. 2008. «Les langues minoritaires en Méditerranée occidentale : entre régression, affirmation et mondialisation ». Revue Dialectologia et Linguistica 16. 81121.

https://www.researchgate.net/profile/Francis_Manzano

Marçais, Philippe. 1977. Esquisse grammaticale de l'arabe maghrébin. Paris : Maisonneuve. 284 pages.

Martin, Jean-François. 1993. Histoire de la Tunisie contemporaine, de Ferry à Bourguiba, 1881-1956. Paris : L'Harmattan. 199 pages.

Marzouki, Samir. 2007. «La francophonie des élites : le cas de la Tunisie ». Revue Hérodote, $\mathrm{n}^{\circ} 126.35-44$.

Memmi, Albert. 1957. Portrait du colonisé, précédé de Portrait du colonisateur. Différentes éditions, dont Petite Bibliothèque Payot, 1973. 180 pages.

Memmi, Albert. 1962. Portrait d'un Juif. Paris : Gallimard. 300 pages. 
Memmi, Albert. 2004. Portrait du décolonisé. Paris : Gallimard. 223 pages.

Naffati, Habiba \& Queffélec, Ambroise. 2004. Le français en Tunisie, [Numéro spécial]. Le Français en Afrique, $\mathrm{n}^{\circ}$ 18. 453 pages.

Sebag, Paul. 1991. Histoire des Juifs de Tunisie. Paris : L'Harmattan. 335 pages.

Soumille, Pierre. 2000. «L'enseignement du français dans la Tunisie du XIX ${ }^{\mathrm{e}}$ siècle avant le Protectorat (1840-1883)». In Dubois Colette, Kasbarian Jean-Michel, Queffélec Ambroise. (dir.), L'expansion du français dans les Suds (XV $-X X^{e}$ siècles), 199-210. Aix-en-Provence: Publications de l'Université de Provence.

Sraieb, Noureddine. 2000. «Place et fonctions de la langue française en Tunisie ». In Dubois Colette, Kasbarian Jean-Michel, Queffélec Ambroise. (dir.), L'expansion du français dans les Suds (XVe-XX siècles), 211-219. Aix-en-Provence : Publications de l'Université de Provence.

Taïeb, Jacques. 2000. Sociétés juives du Maghreb moderne, 1500-1900 : un monde en mouvement. Paris : Maisonneuve et Larose. 223 pages. 